\title{
Skin biopsy as a diagnostic tool in peripheral neuropathy
}

\author{
Giuseppe Lauria* and Grazia Devigili
}

\section{SUMMARY}

Skin biopsy is a safe, minimally invasive, painless and cheap tool for providing diagnostic information on small nerve fibers, which are invisible to routine neurophysiological tests. Biopsy can be performed in hairy skin to investigate unmyelinated and thinly myelinated fibers and in glabrous skin to examine large myelinated fibers. Morphometric analysis of skin nerves is readily accomplished through the use of immunohistochemical techniques, and has proved to be reliable, reproducible and unaffected by the severity of neuropathy. One further advantage of skin biopsy over conventional nerve biopsy is that it allows somatic nerve fibers to be distinguished from autonomic nerve fibers. Morphological changes, axonal degeneration and abnormal regeneration occur in cutaneous nerves very early in the course of peripheral neuropathies, making skin biopsy a promising tool for investigating the progression of neuropathy and the effect of neuroprotective treatments in clinical practice and trials. This article reviews the techniques that are used to investigate the innervation of human skin, the possible uses of skin biopsy in diagnosing and monitoring peripheral neuropathies, and correlations between skin biopsy findings and those of other diagnostic methods.

KEYWORDS autonomic neuropathy, neuropathic pain, peripheral neuropathy, skin biopsy, small-fiber neuropathy

\section{REVIEW CRITERIA}

PubMed was searched using Entrez for articles published up to 4 May 2007, including electronic early release publications. Search terms included "skin biopsy", "neuropathic pain", "small-fiber neuropathy", "autonomic neuropathy", "sensory neuropathy" and "peripheral neuropathy". Full articles were obtained and references were checked for additional material where appropriate.

\section{GME}

G Lauria is a Consultant and Head of the Laboratory for the Study of Cutaneous Innervation at the National Neurological Institute 'Carlo Besta', Milan; G Devigili is a Resident in Neurology and is responsible for the Laboratory for the Study of Cutaneous Innervation at the University of Ferrara, Ferrara, Italy.

\section{Correspondence}

*Neuromuscular Diseases Unit, National Neurological Institute Carlo Besta, Via Celoria 11, 20133 Milan, Italy

glauria@istituto-besta.it

Received 14 May 2007 Accepted 31 July 2007

www.nature.com/clinicalpractice

doi:10.1038/ncpneuro0630
Medscape Continuing Medical Education online Medscape, LLC is pleased to provide online continuing medical education (CME) for this journal article, allowing clinicians the opportunity to earn CME credit. Medscape, LLC is accredited by the Accreditation Council for Continuing Medical Education (ACCME) to provide CME for physicians. Medscape, LLC designates this educational activity for a maximum of 1.0 AMA PRA Category 1 Credits $^{\mathrm{TM}}$. Physicians should only claim credit commensurate with the extent of their participation in the activity. All other clinicians completing this activity will be issued a certificate of participation. To receive credit, please go to http://www.medscape.com/cme/ncp and complete the post-test.

\section{Learning objectives}

Upon completion of this activity, participants should be able to:

1 List advantages of skin biopsy over nerve biopsy.

2 Identify the disadvantage of the blister method compared with the punch method of skin biopsy for neuropathy.

3 List conditions for which immunohistochemical methods have been used to demonstrate small-fiber neuropathy using punch biopsy.

4 Identify systemic diseases in which skin biopsy plays a role in the diagnosis of small-fiber neuropathy and nerve degeneration.

5 Describe conditions under which spontaneous nerve regrowth can be followed using skin biopsy.

\section{INTRODUCTION}

Skin biopsy is proving to be a reliable diagnostic tool in patients complaining of symptoms consistent with small-fiber neuropathy, a condition that has been underdiagnosed in the past. Skin biopsy samples can demonstrate the selective degeneration of somatic unmyelinated fibers that convey pain and thermal sensations. These fibers cannot be observed in routine neurophysiological tests. Skin biopsy can also provide diagnostic information when there is little or no clinical evidence of neuropathy. The minimal invasiveness of skin biopsy makes it a useful tool not only in clinical practice, but also for monitoring the progression of neuropathy in trials of neuroprotective treatments. The range of applications of skin biopsy has recently been expanded to include autonomic neuropathies and immune-mediated and inherited 
demyelinating neuropathies. In addition, correlation of skin biopsy findings with the overall clinical picture and with the results of neurophysiological examinations has provided important insights into the pathogenesis and features of neuropathic pain in peripheral neuropathies. Finally, an extremely interesting field of investigation is that of the functional properties of skin nerves. The emerging view is that the transduction of temperature and pain sensation might be regulated by a network involving the epidermal cells, which are also thought to have a distinct role in the pathogenesis of neuropathic pain.

\section{THE SKIN AND ITS INNERVATION Structure of the superficial skin}

The topmost living layer of the skin is the epidermis, which is composed of four layers of keratinocytes. These keratinocytes undergo gradual differentiation as they progress from the basal layer to the stratum corneum. Epidermal cells have a turnover time of about 30 days. Other cells that reside in the epidermis include Langerhans cells, melanocytes and Merkel cells. The basement membrane (dermal-epidermal junction) separates the epidermis from the subpapillary dermis, which is organized as papillae that contain vascular plexus and capillary loops. In the glabrous skin, the apexes of the papillae contain Meissner's corpuscles. The mean $( \pm S D)$ density of these mechanoreceptors in the fingertip is $33.0 \pm 13.2 / \mathrm{mm}^{2}{ }^{1}{ }^{1}$ Pacini's and Ruffini's corpuscles reside in the deeper layers of the dermis and are commonly excluded from routine skin biopsy examination. The matrix of the superficial dermis also includes fibroblasts, hair follicles, arrectores pilorum muscles, blood vessels, sebaceous glands and sweat glands.

\section{Immunohistochemical examination of skin nerve fibers}

The epidermis is innervated by somatic naked axons arising from nerve bundles that run through the subpapillary dermis. Intraepidermal nerve fibers (IENFs) lose Schwann cell ensheathment as they cross the dermal-epidermal junction and run towards the skin surface between the keratinocytes, sometimes with horizontal branches. In healthy individuals, IENFs have a simple morphology, with small bead-like varicosities. $^{2}$ IENFs can be labeled with antibodies against various structures, including cytoplasmic epitopes (e.g. protein gene product 9.5 [PGP9.5]), components of the cytoskeleton (e.g.
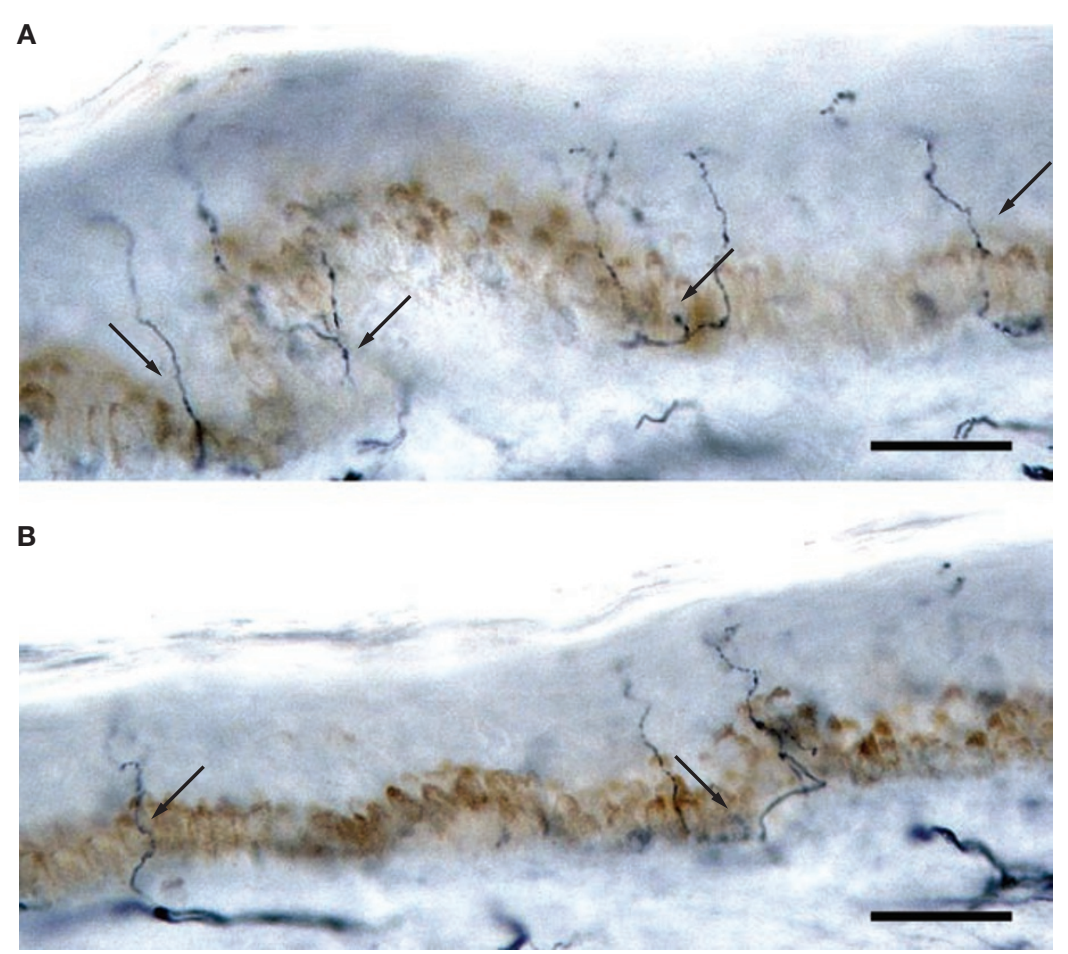

Figure 1 Normal innervation of the epidermis. (A) Proximal thigh. (B) Distal leg. Note the normal morphology of intraepidermal nerve fibers, indicated by the arrows. The fibers have a straight course and slight varicosities. Brightfield immunohistochemistry in $50 \mu \mathrm{m}$ sections stained with polyclonal rabbit anti-protein-gene-product 9.5 antibody (Ultraclone, Wellow, Isle of Wight, UK). Bar $=60 \mu \mathrm{m}$.

microtubules, phosphorylated neurofilaments or axonal membrane), and the capsaicin receptor (transient receptor potential vanilloid receptor 1 [TRPV1]). ${ }^{3}$ The density of IENFs is highest in the paravertebral region of the trunk, and shows a decreasing proximal-to-distal gradient in the limbs, being about $40 \%$ lower in the supramalleolar area than in the thigh (Figure 1).

The subpapillary dermis of both hairy and glabrous skin is innervated by bundles of unmyelinated and thinly myelinated fibers. Glabrous skin also contains several large myelinated fibers, which can be immunostained with antibodies against myelin proteins. ${ }^{1,4}$ Recent studies have demonstrated that myelin ultrastructure and protein expression do not differ between skin and sural nerves. ${ }^{5,6}$

The innervation of dermal autonomic structures, including sweat glands, blood vessels, arrectores pilorum muscles and hair follicles, can be investigated using antibodies against molecules displayed on adrenergic sympathetic fibers (e.g. tyrosine hydroxylase and dopamine beta-hydroxylase), noradrenergic sympathetic 
fibers (e.g. neuropeptide Y), cholinergic sympathetic fibers (e.g. vasointestinal peptide), and vasodilatory peptidergic fibers (e.g. calcitoningene-related peptide [CGRP] and substance $\mathrm{P}$ $[\mathrm{SP}]){ }^{7}$ Skin biopsy, therefore, allows the investigation, separately and specifically, of somatic and autonomic nerve fibers, as well as small-caliber and large-caliber nerve fibers.

\section{Functional properties of skin nerve fibers}

Traditionally, skin nerves have been considered to be transducers of certain sensory stimuli (e.g. temperature, pain and touch) and to have a primary role in maintaining tissue integrity. Recent studies have demonstrated, however, that these nerves are part of a more complex network involving epidermal cells. The emerging view is that keratinocytes and other cells residing in the epidermis communicate with neighboring nerve fibers and might themselves participate in the transduction of certain physical and chemical stimuli. For instance, in vitro studies have shown that mechanical stimulation of keratinocytes can influence the activation of sensory neurons via an intercellular signaling mechanism involving ATP and purinergic receptors. Moreover, keratinocytes secrete chemical substances (e.g. neurotrophins, ATP, $\beta$-endorphin and interleukins) that can influence sensory neurons. ${ }^{8}$ Peptidergic nerves (i.e. those expressing CGRP and SP) influence the maturation of keratinocytes and the ability of Langerhans cells to present antigens to lymphocytes. ${ }^{9,10}$ Neuropeptides released from skin nerves exert various actions on resident cells and blood vessels, resulting in responses such as erythema, edema, hyperthermia and itch. Two groups of CGRP-positive IENFs, projecting to different layers of the dorsal horns, have been described: a large group co-expressing somatostatin, and a smaller group co-expressing SP. Activation of CGRP-somatostatin-positive axons inhibits the activation of CGRP-SP-positive axons during inflammatory responses. ${ }^{11}$ Opioid and N-methyl-D-aspartate (NMDA) receptors have been also identified on skin nerves, suggesting a role for these nerves in neuropathic pain. ${ }^{12,13}$

IENFs are the endings of dorsal root ganglion nociceptors, as demonstrated by their expression of the capsaicin receptor TRPV1, 4,14 a protein that is essential for thermal hyperalgesia induced by tissue inflammation. ${ }^{15}$ Keratinocytes also express receptors of the TRP family, such as TRPV3 and TRPV4, ${ }^{16,17}$ which participate in temperature homeostasis and have a role in the pathogenesis of mechanical hyperalgesia. ${ }^{18}$ Consequently, the whole epidermis can be considered to be a huge polymodal receptor, in which transmission of somatosensory sensation occurs by means of functional contacts between resident cells and nerves. No synaptic contact between keratinocytes and axons has been described, but the loss of Schwann cell ensheathment when IENFs cross the dermalepidermal junction, similar to that seen when large myelinated fibers reach the inner core of mechanoreceptors, ${ }^{19}$ probably favors communication through paracrine pathways.

The active participation of skin cells in the transduction of temperature and pain sensations could explain why IENF density alone does not correlate with the severity of neuropathic pain (see below). In fact, patients with chronic painful neuropathy can show a complete loss of IENFs, suggesting that IENFs are not the generators of pain in these individuals. On the other hand, complete skin denervation is found in patients with insensitivity to pain resulting from hereditary sensory and autonomic neuropathy type IV. ${ }^{20}$ Skin cells could have a role in sensitizing undamaged unmyelinated fibers, in keeping with the concept of 'irritable nociceptors' proposed for postherpetic neuralgia. ${ }^{21}$ Tissue damage could, therefore, lead to widespread functional and degenerative neuropathological changes, as demonstrated in complex regional pain syndrome type I, a condition characterized by a broad spectrum of sensory, autonomic and motor symptoms, but with no apparent evidence of nerve fiber degeneration. ${ }^{22,23}$

\section{SKIN BIOPSY TECHNIQUES}

Skin biopsy is usually performed under topical anesthesia with lidocaine using a sterile disposable $3 \mathrm{~mm}$ punch. The incision does not require suturing, the risk of bleeding and local infection is very low, and no side effects have been reported, even in patients with diabetes. Healing is usually complete within 1 week, and a barely visible scar can remain. For diagnostic purposes in patients with polyneuropathy one skin biopsy sample is taken at the distal end of the leg- $10 \mathrm{~cm}$ above the lateral malleolus - within the territory of the sural nerve. A further biopsy sample can be taken at the proximal thigh $-20 \mathrm{~cm}$ below the iliac spineto demonstrate either the length-dependent process typical of polyneuropathy or the absence 
of a length-dependent pattern, as observed in sensory neuronopathies. One advantage of the skin biopsy method is that its location can be chosen on the basis of the patient's signs and symptoms, and it can be conducted in regions where nerve conduction tests cannot be performed, such as the trunk and fingers.

The 'blister method' is another, even less invasive, method for obtaining samples of epidermis. This technique is performed by applying to the skin surface a suction capsule with single or multiple holes of $2-3 \mathrm{~mm}$ diameter, ${ }^{24}$ which exerts a negative pressure and separates the epidermis from the dermis. This method does not cause bleeding and does not require local anesthesia, and it allows a large area of epidermis to be investigated. The blister method does not, however, provide information on the morphology of IENFs and the innervation of the dermis, so its usefulness in clinical practice is limited.

\section{Quantification of somatic and autonomic skin nerve fibers}

An important advantage of skin biopsy over nerve biopsy is that it allows morphometric analysis of small nerve fibers using bright-field immunohistochemistry or indirect immunofluorescence, rather than relying on morecomplex electron microscope examination. Most laboratories use bright-field microscopy for routine diagnostic purposes (Figure 2). The biopsied tissue is cut into $50 \mu \mathrm{m}$ sections, which are then immunostained with PGP9.5, a neuronal form of ubiquitin carboxyl-terminal hydrolase widely distributed in the PNS. PGP9.5-positive fibers crossing the dermal-epidermal junction are counted in at least three sections. The number of positive fibers is divided by the length of the epidermal surface, calculated using software for biological measurements, to calculate the linear density of IENFs (IENF/mm). Quantification of linear IENF density is a reliable tool, with high interobserver, intraobserver and interlaboratory agreement. ${ }^{25}$ Linear density measurements correlate significantly with data obtained using stereological techniques of skin nerve morphometry, ${ }^{26,27}$ and with quantification of nerve fibers per epidermal area. ${ }^{28}$ No significant variation has been seen in IENF densities calculated in adjacent sections from the same biopsy or in adjacent biopsies from the same site. ${ }^{29}$

Indirect immunofluorescence provides an opportunity to investigate multiple epitopes in the same neural structure or in different structures. The use of confocal laser microscopy allows three-dimensional reconstruction of skin sections, which is particularly useful in the study of cutaneous receptors, sweat glands and blood vessels. Quantification of IENF density is performed using computerized image analysis.

Evaluation of dermal innervation is not currently included in routine morphometric analysis of skin biopsy tissue because of the lack of a validated protocol, although this parameter has recently been measured using the density of PGP9.5-immunoreactive structures and expressed as a percentage of the whole subepidermal area analyzed. ${ }^{30}$ Morphometric data on normal sweat gland innervation are limited, and have been obtained using various methods, including calculation of nerve fiber length ${ }^{31}$ and innervation per unit area. ${ }^{32}$ Other authors have used semiquantitative ${ }^{33}$ or qualitative approaches (Figure 3). ${ }^{34,35}$ Sweat gland denervation and altered expression of neuropeptides were demonstrated in Ross syndrome and familial dysautonomia. ${ }^{33,35}$ In idiopathic pure sudomotor failure, ${ }^{36}$ IENF density and sweat gland innervation are preserved, suggesting that anhidrosis is caused by a functional impairment of cholinergic transmission (V Tugnoli, personal communication). Reduced skin innervation, inversely correlated with the size of blood vessels, has been found in portwine stains, supporting the hypothesis of a causative defect in autonomic vessel innervation. ${ }^{37}$ No study has systematically quantified the innervation of arrectores pilorum muscles, hair follicles and blood vessels.

Using immunohistochemical methods, punch biopsy has also demonstrated small-fiber neuropathy in burning mouth syndrome, ${ }^{38}$ gastric denervation in diabetic patients, ${ }^{39}$ and altered mucosal innervation in rectal hypersensitivity 40 and vulvodynia. ${ }^{41}$

\section{Normative values of intraepidermal nerve fiber density}

Reference ranges of IENF density in the leg are available for both bright-field immunohistochemistry and indirect immunofluorescence techniques. Four studies that investigated IENF density in the distal region of the leg using bright-field immunohistochemistry reported similar results. The mean $( \pm S D)$ linear densities measured in these studies were $12.4 \pm 4.6 \mathrm{IENF} / \mathrm{mm}(n=98),{ }^{29} 13.8$ $\pm 6.7 \mathrm{IENF} / \mathrm{mm}(n=106),{ }^{42} 12.9 \pm 5.3 \mathrm{IENF} / \mathrm{mm}$ 

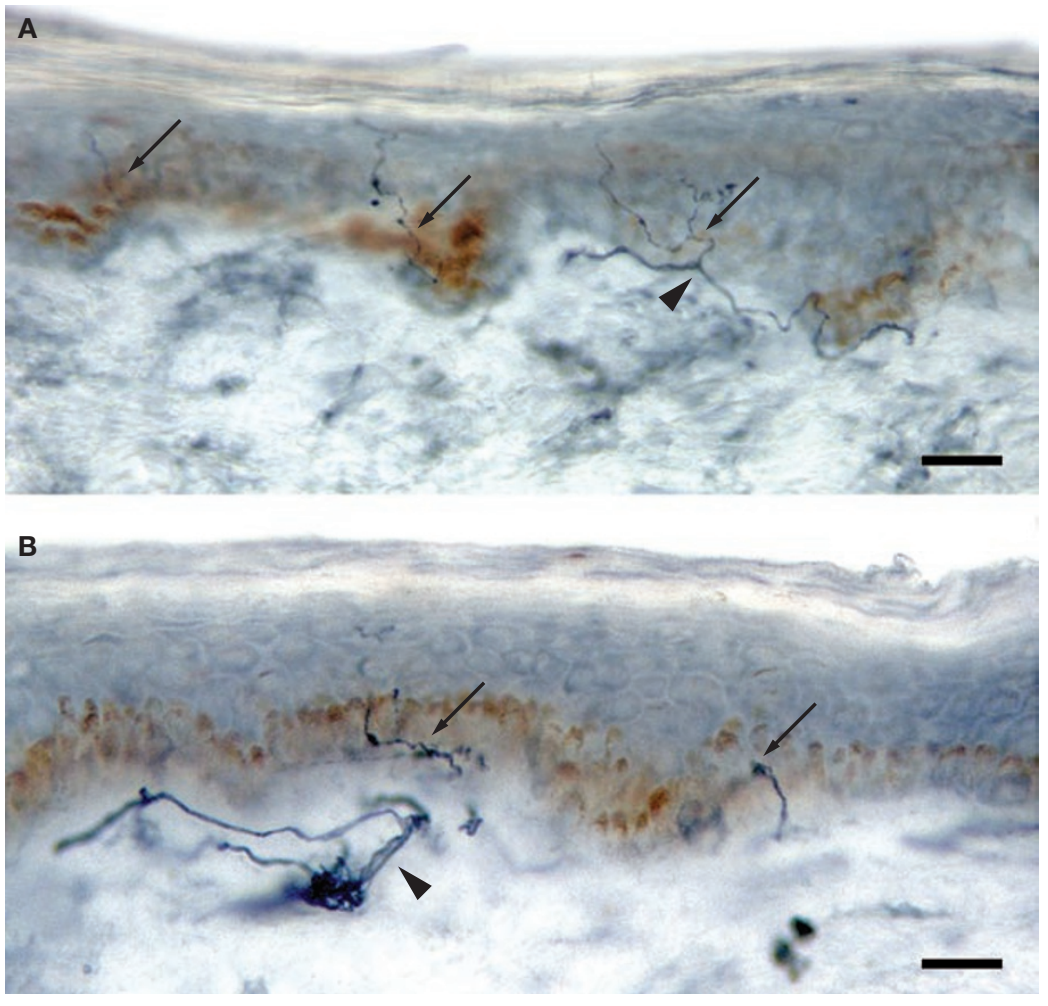

Figure 2 Skin biopsy samples from a patient with diabetic small-fiber neuropathy. (A) Proximal thigh. (B) Distal leg. Arrows indicate intraepidermal nerve fibers, arrowheads indicate dermal nerve bundles. The density of intraepidermal nerve fibers is reduced, particularly in the distal part of the leg, reflecting the length-dependent denervation of the skin. Bright-field immunohistochemistry in $50 \mu \mathrm{m}$ sections stained with polyclonal rabbit antiprotein-gene-product 9.5 antibody (Ultraclone, Wellow, Isle of Wight, UK). Bar $=80 \mu \mathrm{m}$.
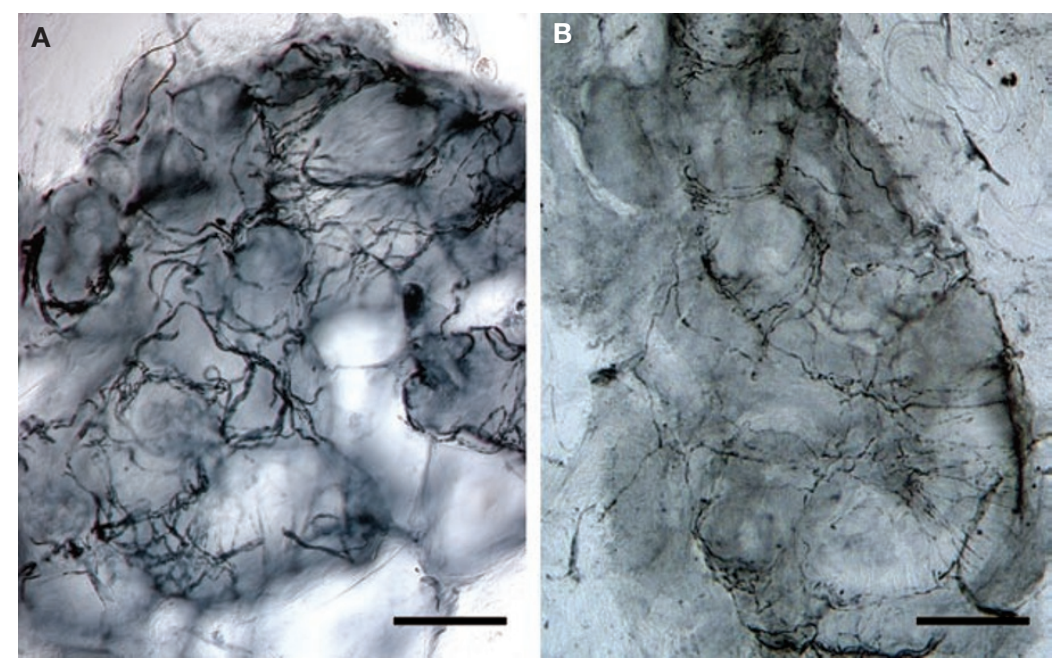

Figure 3 Innervation of the sweat gland. (A) Normal innervation in a healthy subject. (B) Reduced density of nerve fibers in a patient with peripheral neuropathy. Bright-field immunohistochemistry in $50 \mu \mathrm{m}$ sections stained with polyclonal rabbit anti-protein-gene-product 9.5 antibody (Ultraclone, Wellow, Isle of Wight, UK). Bar $=80 \mu \mathrm{m}$. $(n=55),{ }^{43}$ and $15.0 \pm 5.0 \mathrm{IENF} / \mathrm{mm}(n=84) .{ }^{44}$ Two of these studies also reported the IENF density in the proximal thigh, as $21.1 \pm 10.4 \mathrm{IENF} / \mathrm{mm}^{29}$ and $22.8 \pm 6.9 \mathrm{IENF} / \mathrm{mm}^{44}$ Several smaller studies ${ }^{45-49}$ have reported similar values. Indirect immunofluorescence (with or without confocal microscopy) has been used in fewer studies, none of which was specifically designed to assess the normative range of IENF density. Values reported for the distal region of the leg were higher than those found using bright-field immunohistochemistry, ranging between $17.4 \pm 7.4 / \mathrm{mm}$ and $33.0 \pm 7.9 / \mathrm{mm}^{50-53}$

The normal IENF density in the arm was investigated in two studies that used different techniques. Pan and colleagues ${ }^{43}$ found a density of $17.3 \pm 6.2 \mathrm{IENF} / \mathrm{mm}$ in the distal forearm using bright-field immunohistochemistry, whereas Nolano and colleagues ${ }^{1}$ estimated a density of $11.3 \pm 2.9 \mathrm{IENF} / \mathrm{mm}$ in the glabrous skin of the fingertip using confocal microscopy.

The effect of aging on skin innervation remains uncertain. In the first normative study performed at the Johns Hopkins University, Baltimore, $\mathrm{MD},{ }^{29}$ sex and age did not affect IENF density in the distal part of the leg. Similarly, two studies showed that IENF density in the fingertip ${ }^{1}$ and abdominal skin ${ }^{54}$ did not change with age. Aging did, however, correlate with decreasing IENF density in the distal part of the leg in studies performed in Norway and Singapore. ${ }^{42,44}$ Researchers in Taiwan found a difference in density in the distal part of the leg between subjects aged below 60 years ( 11.1 $\pm 3.7 \mathrm{IENF} / \mathrm{mm}$ ) and those over 60 years of age $(7.6 \pm 3.0 \mathrm{IENF} / \mathrm{mm}) .{ }^{43,55,56}$ No other anthropometric variable influences IENF density in the distal part of the leg, ${ }^{44}$ although male gender was associated with lower values in one study. ${ }^{42}$

\section{DIAGNOSTIC YIELD OF SKIN BIOPSY IN SMALL-FIBER NEUROPATHY}

In 2005, a task force of the European Federation of Neurological Societies published guidelines on the use of skin biopsy in the diagnosis of peripheral neuropathies, in which the usefulness of the technique was established. ${ }^{57}$ IENF density in the distal part of the leg has been found to be useful for confirming a diagnosis of peripheral neuropathy of various etiologies with specificity ranging from $95 \%{ }^{55}$ to $97 \%, 29$ sensitivity ranging from $45 \%{ }^{29}$ to $80 \%,{ }^{55}$ a positive predictive value of $92 \%$, and a negative predictive value of $90 \% .{ }^{29}$ Since 2005 , other 
papers, focused on pure small-fiber neuropathy, have provided additional support for the important role of skin biopsy in diagnosis. ${ }^{58-61}$ Smallfiber neuropathy is frequently encountered in clinical practice, but can be misdiagnosed because of the absence of neurophysiological tests that can investigate small nerve fibers. In most studies, patients' complaints-mainly burning feet-are taken as the gold standard against which the performance of skin biopsy is measured. Studies in patients with clinically suspected pure small-fiber neuropathy reported that skin biopsy had a sensitivity of $90 \%$ and a specificity of $95 \% .25,55$ In idiopathic and secondary (diabetic, cytotoxic or amyloid) smallfiber neuropathy, skin biopsy analysis showed a positive predictive value of $95 \%$ and a negative predictive value of $91 \% .{ }^{28}$ Most recently, a study compared 99 patients who had painful neuropathy with 37 healthy subjects and assessed sensitivity and specificity of both IENF and dermal nerve fiber density quantification using receiver operating characteristic analysis. ${ }^{30}$ In patients with pure small-fiber neuropathy, using a cut-off of $8.8 \mathrm{IENF} / \mathrm{mm}$ in the distal region of the leg, sensitivity of IENF density was $77 \%$ and specificity was 79\%. Quantification of dermal nerve fiber density in addition to IENF density increased the sensitivity to $86 \%$. IENF quantification is highly reproducible and its reliability as a diagnostic tool is not affected by the severity of the neuropathy. ${ }^{29}$ Several smaller studies ${ }^{45-53,62-69}$ involving a total of more than 500 patients have confirmed these data, demonstrating an important diagnostic role for skin biopsy in patients who have little clinical and neurophysiological evidence of neuropathy.

\section{MORPHOLOGICAL CHANGES IN SKIN NERVES IN NEUROPATHIES}

The presence of diffuse swellings on IENFs (Figure 4) has been shown to predict the progression to overt neuropathy in patients with HIV, diabetes or other causes of smallfiber neuropathy, ${ }^{47,60,69}$ and to correlate with paresthesia. ${ }^{60}$ Swelling was defined either quantitatively (e.g. enlargement above $1.5 \mu \mathrm{m})^{47}$ or semi-quantitatively (e.g. enlargements to more than twice the diameter of the parent fiber). ${ }^{60,69}$ Although swellings are commonly considered to be predegenerative changes, they can also reflect axonal regeneration, as has been observed after capsaicin denervation ${ }^{70}$ or steroid treatment. ${ }^{71}$

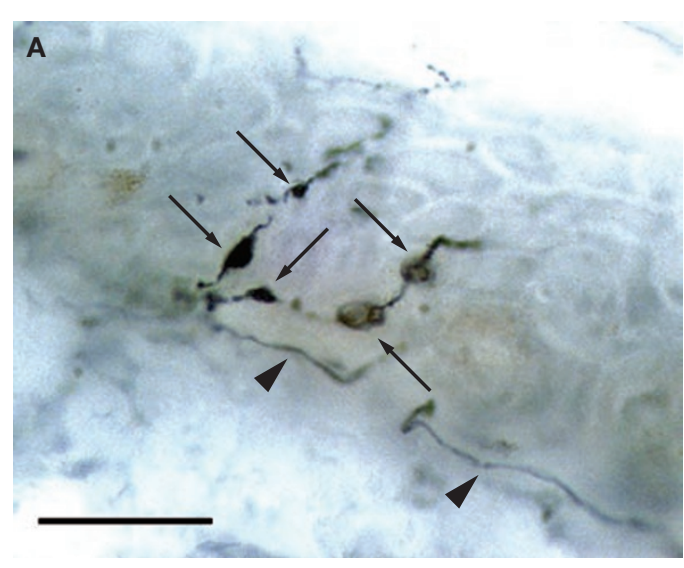

Figure 4 Skin biopsy tissue from the distal part of the leg in a patient with peripheral neuropathy. Arrows indicate the multiple swellings, some of them partly vacuolated, of an intraepidermal nerve fiber branching above the dermalepidermal junction. Arrowheads indicate the nerves in the subpapillary dermis. Bright-field immunohistochemistry in $50 \mu \mathrm{m}$ sections stained with polyclonal rabbit anti-protein-gene-product 9.5 antibody (Ultraclone, Wellow, Isle of Wight, UK). Bar $=50 \mu \mathrm{m}$.

Increased branching and sprouting of IENFs was found more frequently in patients with neuropathy than in healthy controls, and might represent an early marker of nerve fiber dysfunction. ${ }^{2,72}$ We do not know, however, whether collateral sprouting represents attempted axonal regeneration with the goal of achieving functional recovery of nerve fibers. ${ }^{73,74}$

\section{CORRELATES OF INTRAEPIDERMAL NERVE FIBER DENSITY Clinical picture, etiology and neuropathic pain}

The clinical picture of small-fiber neuropathy is dominated by spontaneous and stimulus-evoked positive sensory symptoms-namely thermal and pinprick hypoesthesia-that can mask the signs of small-fiber loss. Only a few studies have attempted to correlate IENF density with validated clinical scales. In patients with diabetic neuropathy, a negative correlation between IENF density and neuropathy symptom score was reported. ${ }^{53,56}$ These studies also showed that the extent of epidermal denervation correlated with the duration of diabetes but not with hemoglobin $A_{1 C}$ levels, suggesting that IENF density might be useful as a marker of neuropathy progression. A recent study found a 
high concordance between reduced IENF density and loss of pinprick sensation in the foot. ${ }^{61}$

Skin biopsy has allowed small-fiber neuropathy to be demonstrated in restless legs syndrome ${ }^{75}$ and erythromelalgia. ${ }^{76}$ In systemic diseases, such as systemic lupus erythematosus, sarcoidosis, Sjögren's syndrome, celiac disease and hypothyroidism, skin biopsy has enabled correlations to be found between neuropathic symptoms and small-fiber degeneration. ${ }^{52,65,77-79}$ Although IENF density is a general marker of axonal integrity in peripheral neuropathies, it cannot be used to directly address the question of etiology. Skin biopsy findings can, however, indirectly contribute to the assessment of etiology. For example, in $40 \%$ of patients with small-fiber neuropathy diagnosed only after skin biopsy, oral glucose tolerance testing revealed a previously undetected impaired glucose tolerance. ${ }^{49}$ Similarly, the distribution of IENF loss can help to differentiate between a non-length-dependent sensory neuronopathy and a length-dependent axonal neuropathy, ${ }^{78,80}$ thereby leading to focused screening for associated diseases.

The relationship between IENF density and neuropathic pain remains uncertain. In HIV neuropathy, IENF density correlated inversely with pain severity when assessed by the patient, but not when the Gracely Pain Scale was used. ${ }^{66}$ Another study found only a trend towards an inverse correlation between IENF density and pain intensity in this setting. ${ }^{81}$ In diabetic neuropathy, patients with pain had lower IENF densities than did asymptomatic patients, but IENF density did not correlate with pain intensity within the group of symptomatic patients. ${ }^{82}$ In patients with impaired glucose tolerance, diet and exercise induced a slight recovery of IENF density that was associated with a reduction in pain symptoms. ${ }^{83}$ Similarly, epidermal reinnervation coincided with pain reduction after steroid treatment. ${ }^{71}$ In length-dependent neuropathies, therefore, more-severe IENF loss seems to increase the risk of developing pain, the intensity of which might decrease in parallel with recovery of IENF density.

In postherpetic neuralgia, on the basis of evidence of relatively preserved skin innervation in the area of severe allodynia, normal thermal sensory function, pain relief in response to topical lidocaine, and worsening of pain with application of capsaicin, surgical removal of painful skin has been attempted. ${ }^{84}$
After initial relief, pain increased, became intractable, and spread to previously unaffected dermatomes, suggesting the involvement of central mechanisms in the pathogenesis of neuropathic pain.

\section{Sensory nerve conduction studies}

Sural sensory nerve action potential (SNAP) amplitude, which reflects the integrity of largediameter fibers, showed concordance with IENF density in the distal part of the leg in patients with large-fiber or mixed small-fiber and largefiber neuropathy. Not surprisingly, skin biopsy analysis seemed to be more sensitive than sural nerve conduction studies for diagnosing smallfiber neuropathy. ${ }^{62}$ One study, ${ }^{85}$ however, showed that in patients with symptoms of small-fiber neuropathy and normal sural nerve conduction, reduced IENF density correlated with a decrease in SNAP amplitude in the medial plantar nerve. This finding suggests subclinical involvement of the most-distal large fibers in small-fiber neuropathy.

\section{Psychophysical tests}

The detection of thermal and pain thresholds using quantitative sensory testing has been widely used to assess the function of small nerve fibers. Although this approach is useful in population studies, it is an unreliable tool for diagnosing small-fiber neuropathy in clinical practice. ${ }^{86}$ Moreover, the size of the probe used for the test can affect the results. ${ }^{87}$

In view of the fact that unmyelinated fibers and thinly myelinated fibers convey warm and cold sensation, respectively, thermal thresholds would be expected to correlate with IENF density. In diabetic neuropathy, IENF density was found to be inversely correlated with thermal and pain thresholds, showing the highest correlation with warm threshold. $.33,56,82$ Similarly, in Guillain-Barré syndrome lower IENF density was associated with increased warm threshold. ${ }^{67}$ One study reported a significant correlation between cold pain threshold and signs of large-fiber impairment. ${ }^{59}$ By contrast, others studies did not find any correlation between quantitative sensory testing results and IENF density. ${ }^{45,51,88}$

\section{Autonomic tests}

As IENFs are somatic unmyelinated fibers, their density would not be expected to correlate with autonomic fiber function. Intriguingly, however, 
in patients with Guillain-Barré syndrome and chronic inflammatory demyelinating polyradiculoneuropathy, lower IENF density was associated with a higher risk of developing dysautonomia. ${ }^{64,67}$ These findings suggest that the integrity of IENFs might reflect the integrity of the whole class of small nerve fibers, including autonomic fibers. A few studies have investigated the correlation between IENF density and the results of a quantitative sudomotor axonal reflex test in patients with painful neuropathy and autonomic symptoms in order to test the hypothesis that IENF density and sweat output might decrease concomitantly. IENF density correlated with test results in one study, ${ }^{63}$ but not in another. ${ }^{51}$ In leprosy neuropathy, reduced nicotine-induced axon-reflex sweating correlated with decreased innervation of sweat glands. ${ }^{88}$

\section{Nonconventional neurophysiological tests}

Laser-evoked potentials (LEPs) have been used to investigate peripheral and central nociceptive pathways in trigeminal neuralgia and peripheral neuropathies. Late LEPs, reflecting A $\delta$-fiber activation, are delayed in patients with neuropathic pain, but can be enhanced when the pain has a psychogenic origin. ${ }^{89}$ Recording of ultralate LEPs, reflecting activation of unmyelinated C-fibers, is less reliable than recording of late LEPs, thereby limiting the overall usefulness of LEPs in clinical practice. LEPs and skin biopsy findings have been examined in single case reports. ${ }^{90}$ In two patients with Ross syndrome, abnormal LEPs correlated with decreased IENF density and increased thermal thresholds. ${ }^{91}$ No study has yet looked for a correlation between results of skin biopsy analysis and recording of contact heat-evoked potentials, a technique that was recently proposed for investigating smallfiber function, but which cannot be used to assess C-fiber-related responses. ${ }^{92}$

Microneurography allows single-fiber recordings from nerves in awake patients. This technique demonstrated loss of nociceptive and skin sympathetic C-fiber activity that correlated with IENF and sweat gland denervation in a patient with hereditary sensory and autonomic neuropathy type IV. ${ }^{20}$ In two patients with generalized anhidrosis, C-fiber recording and sweat gland innervation analysis distinguished postganglionic autonomic nerve fiber impairment from eccrine gland dysfunction. ${ }^{34}$

\section{Sural nerve biopsy}

The diagnosis of small-fiber neuropathy is better assessed by skin biopsy than by sural nerve biopsy. ${ }^{57}$ IENF density can be reduced despite normal morphometry of unmyelinated and thinly myelinated fibers in sural nerve biopsy. ${ }^{58}$ In a large comparative study, ${ }^{62}$ skin and sural nerve biopsy findings were concordant in $73 \%$ of patients, but in $23 \%$ of patients IENF density was the only indicator of small-fiber neuropathy. Skin biopsy offers the opportunity to differentiate small nerve fibers with somatic function from those with autonomic function, thereby giving it a further advantage over nerve biopsy. In Charcot-Marie-Tooth disease and related hereditary neuropathies, a biopsy sample of the glabrous skin demonstrated the typical neuropathological abnormalities known from sural nerve studies. ${ }^{5,6}$

Immunohistochemical studies demonstrated IgM deposited specifically in the myelinated fibers of hairy and glabrous skin in patients with anti-myelin-associated-glycoprotein neuropathy. ${ }^{93}$ Although skin biopsy can be contemplated in genetic and immune-mediated neuropathies, sural nerve biopsy should always be considered to confirm the diagnosis in inflammatory polyradiculoneuropathy with atypical presentation, or when vasculitic or amyloid neuropathy is suspected.

\section{MONITORING THE PROGRESSION OF NEUROPATHY WITH SKIN BIOPSY}

Skin biopsy is minimally invasive and can be repeated close to the site of a previous biopsy, within the territory of the same sensory nerve, to investigate the progression of neuropathy and the effect of neuroprotective treatments. IENFs can spontaneously regenerate, in parallel with sensory recovery, after nerve injury; for example, in diabetic truncal neuropathy, ${ }^{94}$ following chemical denervation with capsaicin, ${ }^{70}$ or after intracutaneous axotomy. ${ }^{73}$ The ability of skin nerves to regenerate after topical capsaicin treatment prompted study of the rate of fiber regrowth in patients at risk of developing neuropathy. In diabetic or HIV-infected patients with no clinical or neurophysiological signs of neuropathy, the IENF regeneration rate is slower than in healthy subjects. ${ }^{73,95}$ This finding provided insights into the pathogenesis of these common neuropathies and suggested the need for early neuroprotective interventions. In patients with impaired glucose tolerance, drastic 
www.nature.com/clinicalpractice/neuro
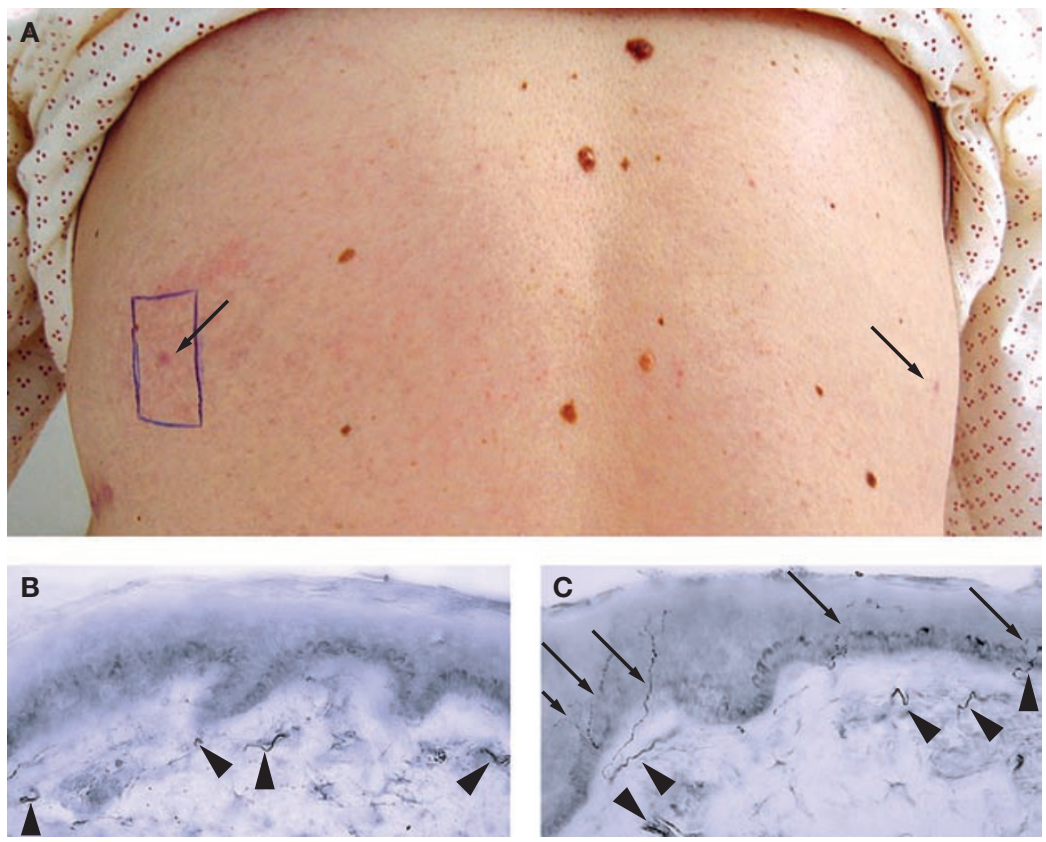

Figure 5 Skin biopsy in notalgia paresthetica. (A) Biopsies were performed within an area of notalgia paresthetica, indicated by the blue square on the left side of the trunk, and on the healthy contralateral dermatome. Arrows indicate the scars 2 weeks after biopsy. (B) Note the complete loss of intraepidermal nerve fibers and the denervation of the dermis in the pathological area. (C) Normally innervated skin in the healthy area for comparison. Arrows indicate intraepidermal nerve fibers, arrowheads indicate dermal nerve bundles. Bright-field immunohistochemistry in $50 \mu \mathrm{m}$ sections stained with polyclonal rabbit anti-protein-gene-product 9.5 antibody (Ultraclone, Wellow, Isle of Wight, UK). Bar $=60 \mu \mathrm{m}$.

\section{CONCLUSIONS: USEFULNESS AND LIMITATIONS OF SKIN BIOPSY}

Skin biopsy should be considered in patients with symptoms of small-fiber neuropathy when nerve conduction studies do not reveal abnormalities. Innervation density should be assessed using a standardized quantitative method, the protocol for which has been recently published. ${ }^{104}$ Qualitative examination of epidermal innervation density could be misleading, and should not be used alone to diagnose small-fiber neuropathy. Once smallfiber neuropathy has been diagnosed, focused screening (e.g. glucose tolerance test) and treatment of neuropathic pain can begin. Skin biopsy provides an opportunity to identify subclinical involvement of autonomic nerve fibers and degeneration of somatic nerves in neuropathies otherwise considered exclusively autonomic, leading to a better comprehension of symptoms and awareness of potential complications.

Skin biopsy allows preganglionic injury (e.g. radiculopathy), in which nerve fiber density is preserved, to be differentiated from postganglionic damage (e.g. plexopathy and neuropathy), in which nerve fibers degenerate. The technique can be useful when painful symptoms are localized in the trunk (e.g. notalgia paresthetica; Figure 5) or proximal regions of the limbs (e.g. meralgia paresthetica), where sensory nerve conduction cannot be recorded. Despite high specificity, sensitivity and positive and negative predictive values, some patients with neuropathy can show normal IENF density. In patients with typical length-dependent distribution of symptoms, however, predegenerative changes of skin nerves, including axonal swellings and weaker PGP9.5 immunoreactivity, are commonly found, and a follow-up biopsy should be performed to evaluate the progression of the neuropathy. IENF density within the normal range of values can be found in patients with demyelinating neuropathy with predominant large-fiber impairment.

One limitation of skin biopsy is that it cannot help in assessment of the etiology of neuropathy. The technique cannot replace nerve biopsy when neuropathological examination of mixed or large-fiber neuropathy is needed and when a vasculitic pathogenesis is suspected. Finally, despite high positive and negative predictive values in small-fiber neuropathy, normal IENF density cannot exclude a functional impairment of unmyelinated fibers. 


\section{KEY POINTS}

- Skin biopsy analysis is a reliable tool for diagnosing small-fiber neuropathy

- Skin biopsy is a safe and painless procedure that allows somatic fibers carrying temperature and pain sensation to be differentiated from autonomic fibers

- Biopsy of glabrous skin can be performed to examine large sensory fibers in immunemediated and inherited demyelinating neuropathies

- Loss of intraepidermal nerve fibers correlates with increased severity of neuropathy and a higher risk of developing neuropathic pain

- Skin nerve fibers are able to regenerate, and their regrowth rate could be a marker of early axonal damage in patients at risk of developing peripheral neuropathy

- $\quad$ Skin biopsy can be repeated in close proximity to a previous biopsy in order to assess the progression of neuropathy and the response to neuroprotective treatments, and can be used as a measure of outcome in clinical trials

\section{References}

1 Nolano M et al. (2003) Quantification of myelinated endings and mechanoreceptors in human digital skin. Ann Neurol 54: 197-205

2 Wendelschafer-Crabb G et al. (2006) Morphological features of nerves in skin biopsies. J Neurol Sci 242: $15-21$

3 Lauria G et al. (2006) Expression of capsaicin receptor immunoreactivity in human peripheral nervous system and in painful neuropathies. J Peripher Nerv Syst 11: 262-271

4 Lauria G et al. (2004) Tubule and neurofilament immunoreactivity in human hairy skin: markers for intraepidermal nerve fibers. Muscle Nerve 30: 310-316

5 Li J et al. (2005) Skin biopsies in myelin-related neuropathies: bringing molecular pathology to the bedside. Brain 128: 1168-1177

6 Sabet A et al. (2006) Skin biopsies demonstrate MPZ splicing abnormalities in Charcot-Marie-Tooth neuropathy 1B. Neurology 67: 1141-1146

7 Albrecht PJ et al. (2006) Pathologic alterations of cutaneous innervation and vasculature in affected limbs from patients with complex regional pain syndrome. Pain 120: 244-266

8 Lumpkin EA and Caterina MJ (2007) Mechanisms of sensory transduction in the skin. Nature 445: 858-865

9 Beresford L et al. (2004) Nerve fibres are required to evoke a contact sensitivity response in mice. Immunology 111: 118-125

10 Hosoi J et al. (1993) Regulation of Langerhans cell function by nerves containing calcitonin gene-related peptide. Nature 363: 159-163

11 Gibbins IL et al. (1987) Two immunohistochemically identified populations of calcitonin gene-related peptide (CGRP)-immunoreactive axons in human skin. Brain Res 414: 143-148

12 Ständer S et al. (2002) Localization of $\mu$-opioid receptor $1 \mathrm{~A}$ on sensory nerve fibers in human skin. Regul Pept 110: 75-83
13 Kinkelin I et al. (2000) Localization of ionotropic glutamate receptors in peripheral axons of human skin. Neurosci Lett 283: 149-152

14 Ständer S et al. (2004) Expression of vanilloid receptor subtype 1 in cutaneous sensory nerve fibers, mast cells, and epithelial cells of appendage structures. Exp Dermatol 13: 129-139

15 Davis JB et al. (2000) Vanilloid receptor-1 is essential for inflammatory thermal hyperalgesia. Nature 405 : 183-187

16 Peier AM et al. (2002) A heat-sensitive TRP channel expressed in keratinocytes. Science 296: 2046-2049

17 Smith GD et al. (2002) TRPV3 is a temperaturesensitive vanilloid receptor-like protein. Nature 418: 186-190

18 Suzuki Y et al. (2002) Lowered response threshold and increased responsiveness to mechanical stimulation of cutaneous nociceptive fibers in streptozotocin-diabetic rat skin in vitro-correlates of mechanical allodynia and hyperalgesia observed in the early stage of diabetes. Neurosci Res 43: $171-178$

19 Johnson KO (2001) The roles and functions of cutaneous mechanoreceptors. Curr Op Neurobiol 11: 455-461

20 Nolano M et al. (2000) Absent innervation of skin and sweat glands in congenital insensitivity to pain with anhidrosis. Clin Neurophysiol 111: 1596-1601

21 Oaklander AL (2001) The density of remaining nerve endings in human skin with and without postherpetic neuralgia after shingles. Pain 92: 139-145

22 Albrecht PJ et al. (2006) Pathologic alterations of cutaneous innervation and vasculature in affected limbs from patients with complex regional pain syndrome. Pain 120: 244-266

23 Oaklander AL et al. (2006) Evidence of focal smallfiber axonal degeneration in complex regional pain syndrome-I (reflex sympathetic dystrophy). Pain 120: 235-243

24 Kennedy WR et al. (1999) A skin blister method to study epidermal nerves in peripheral nerve disease. Muscle Nerve 22: 360-371

25 Smith AG et al. (2005) The reliability of skin biopsy with measurement of intraepidermal nerve fiber density. J Neurol Sci 228: 65-69

26 Stocks EA et al. (1996) An unbiased method for estimation of total epidermal nerve fibre length. J Neurocytol 25: 637-644

27 Hilliges $M$ and Johansson O (1999) Comparative analysis of numerical estimation methods of epithelial nerve fibers using tissue sections. J Peripher Nerv Syst 4: 53-57

28 Koskinen $\mathrm{M}$ et al. (2005) A quantitative method for the assessment of intraepidermal nerve fibers in smallfiber neuropathy. J Neurol 252: 789-794

29 McArthur JC et al. (1998) Epidermal nerve fiber density: normative reference range and diagnostic efficiency. Arch Neurol 55: 1513-1520

30 Vlčková-Moravcová E et al.: Diagnostic validity of skin biopsy, utilising intra- and subepidermal nerve fibre densities in painful sensory neuropathies. Muscle Nerve [doi:10.1002/mus.20889]

31 Hirai A et al. (2000) Evaluation of diabetic neuropathy through the quantitation of cutaneous nerves. J Neurol Sci 172: 55-62

32 Sommer C et al. (2002) Selective loss of cholinergic sudomotor fibers causes anhidrosis in Ross syndrome. Ann Neurol 52: 247-250

33 Hilz MJ et al. (2004) Assessing function and pathology in familial dysautonomia: assessment of temperature perception, sweating and cutaneous innervation. Brain 127: 2090-2098 
34 Donadio V et al. (2005) Generalised anhidrosis: different lesion sites demonstrated by microneurography and skin biopsy. J Neurol Neurosurg Psychiatry 76: 588-591

35 Nolano M et al. (2006) Ross syndrome: a rare or a misknown disorder of thermoregulation? A skin innervation study on 12 subjects. Brain 129: 2119-2131

36 Nakazato Y et al. (2004) Idiopathic pure sudomotor failure: anhidrosis due to deficits in cholinergic transmission. Neurology 63: 1476-1480

37 Selim MM et al. (2004) Confocal microscopy study of nerves and blood vessels in untreated and treated port wine stains: preliminary observations. Dermatol Surg 30: 892-897

38 Lauria G et al. (2005) Trigeminal small-fiber sensory neuropathy causes burning mouth syndrome. Pain 115: 332-337

39 Kennedy WR (2004) Opportunities afforded by the study of unmyelinated nerves in skin and other organs. Muscle Nerve 29: 756-767

40 Chan CLH et al. (2003) Sensory fibers expressing capsaicin receptor TRPV1 in patients with rectal hypersensitivity and faecal ungency. Lancet 361 : 385-391

41 Tympanidis P et al. (2003) Increased innervation of the vulval vestibule in patients with vulvodynia. $\mathrm{Br} J$ Dermatol 148: 1021-1027

42 Gøransson LG et al. (2004) The effect of age and gender on epidermal nerve fiber density. Neurology 62: 774-777

43 Pan CL et al. (2001) Degeneration of nociceptive nerve terminals in human peripheral neuropathy. Neuroreport 12: 787-792

44 Umapathi T et al. (2006) Determinants of epiderma nerve fiber density in normal individuals. Muscle Nerve 33: 742-746

45 Holland NR et al. (1997) Intraepidermal nerve fiber density in patients with painful sensory neuropathy. Neurology 48: 708-711

46 Lauria G et al. (1999) Epidermal innervation: changes with aging, topographic location, and in sensory neuropathy. J Neurol Sci 164: 172-178

47 Lauria G et al. (2003) Axonal swellings predict the degeneration of epidermal nerve fibers in painful neuropathies. Neurology 61: 631-636

48 Scott LJ et al. (1999) Quantitative analysis of epidermal innervation in Fabry disease. Neurology 52: 1249-1254

49 Smith AG et al. (2001) Epidermal nerve innervation in impaired glucose tolerance and diabetes-associated neuropathy. Neurology 57: 1701-1704

50 Kennedy WR et al. (1996) Quantitation of epidermal nerves in diabetic neuropathy. Neurology 47: 1042-1048

51 Periquet Ml et al. (1999) Painful sensory neuropathy: prospective evaluation using skin biopsy. Neurology 53: 1641-1647

52 Hoitsma E et al. (2002) Small fibre neuropathy in sarcoidosis. Lancet 359: 2085-2086.

53 Pittenger GL et al. (2004) Intraepidermal nerve fibers are indicators of small-fiber neuropathy in both diabetic and nondiabetic patients. Diabetes Care 27: 1974-1979

54 Besne l et al. (2002) Effect of age and anatomical site on density of sensory innervation in human epidermis. Arch Dermatol 138: 1445-1450

55 Chien HF et al. (2001) Quantitative pathology of cutaneous nerve terminal degeneration in the human skin. Acta Neuropathol 102: 455-461

56 Shun CT et al. (2004) Skin denervation in type 2 diabetes: correlations with diabetic duration and functional impairments. Brain 127: 1593-1605
57 Lauria G et al. (2005) EFNS guidelines on the use of skin biopsy in the diagnosis of peripheral neuropathy. Eur J Neurol 12: 747-758

58 Holland NR et al. (1998) Small-fiber sensory neuropathies: clinical course and neuropathology of idiopathic cases. Ann Neurol 44: 47-59

59 Loseth S et al. (2006) Intraepidermal nerve fibre density, quantitative sensory testing and nerve conduction studies in a patient material with symptoms and signs of sensory polyneuropathy. Eur J Neurol 13: 105-111

60 Gibbons $\mathrm{CH}$ et al. (2006) The utility of skin biopsy for prediction of progression in suspected small fiber neuropathy. Neurology 66: 256-258

61 Walk D et al. (2007) Concordance between epidermal nerve fiber density and sensory examination in patients with symptoms of idiopathic small fiber neuropathy. J Neurol Sci. 255: 23-26

62 Herrmann DN et al. (1999) Epidermal nerve fiber density and sural nerve morphometry in periphera neuropathies. Neurology 53: 1634-1640

63 Novak V et al. (2001) Autonomic impairment in painful neuropathy. Neurology 56: 861-868

64 Chiang MC et al. (2002) Cutaneous innervation in chronic inflammatory demyelinating polyneuropathy. Neurology 59: 1094-1098

65 Omdal R et al. (2002) Small nerve fiber involvement in systemic lupus erythematosus: a controlled study. Arthritis Rheum 46: 1228-1232

66 Polydefkis M et al. (2002) Reduced intraepiderma nerve fiber density in HIV-associated sensory neuropathy. Neurology 58: 115-119

67 Pan CL et al. (2003) Cutaneous innervation in GuillainBarré syndrome: pathology and clinical correlations. Brain 126: 386-397

68 Sumner CJ et al. (2003) The spectrum of neuropathy in diabetes and impaired glucose tolerance. Neurology 60: 108-111

69 Herrmann DN et al. (2004) Epidermal nerve fiber density, axonal swellings and QST as predictors of HIV distal sensory neuropathy. Muscle Nerve 29: 420-427

70 Nolano M et al. (1999) Topical capsaicin in humans: parallel loss of epidermal nerve fibers and pain sensation. Pain 81: 135-145

71 Nodera $\mathrm{H}$ et al. (2003) Epidermal reinnervation concomitant with symptomatic improvement in a sensory neuropathy. Muscle Nerve 27: 507-509

72 Lauria G et al. (1999) Epidermal innervation: changes with aging, topographic location, and sensory neuropathy. J Neurol Sci 164: 172-178

73 Hahn Ket al. (2007) Impaired reinnervation in HIV infection following experimental denervation. Neurology 68: 1251-1256

74 Hahn Ket al. (2006) Collateral sprouting of human epidermal nerve fibers following intracutaneous axotomy. J Peripher Nerv Syst 11: 142-147

75 Polydefkis M et al. (2000) Subclinical sensory neuropathy in late-onset restless legs syndrome. Neurology 55: 1115-1121

76 Davis MD et al. (2006) Histopathologic findings in primary erythromelalgia are nonspecific: special studies show a decrease in small nerve fiber density. J Am Acad Dermatol 55: 519-522

77 Ørstavik Ket al. (2006) Pain and small-fiber neuropathy in patients with hypothyroidism. Neurology 67: 786-791

78 Brannagan TH III et al. (2005) Small-fiber neuropathy/neuronopathy associated with celiac disease: skin biopsy findings. Arch Neurol 62: 1574-1578

79 Chai J et al. (2005) Painful small-fiber neuropathy in Sjögren syndrome. Neurology 65: 925-927 
80 Sghirlanzoni A et al. (2005) Sensory neuron diseases. Lancet Neurol 4: 349-361

81 Herrmann DN et al. (2006) Is skin biopsy a predictor of transition to symptomatic HIV neuropathy? A longitudinal study. Neurology 66: 857-861

82 Sorensen Let al. (2006) The relationship among pain, sensory loss, and small nerve fibers in diabetes. Diabetes Care 29: 883-887

83 Smith AG et al. (2006) Lifestyle intervention for prediabetic neuropathy. Diabetes Care 29: 1294-1299

84 Petersen KL and Rowbotham MC (2007) Relief of post-herpetic neuralgia by surgical removal of painful skin: 5 years later. Pain 131: 214-218

85 Herrmann DN et al. (2004) Plantar nerve AP and skin biopsy in sensory neuropathies with normal routine conduction studies. Neurology 63: 879-885

86 Shy ME et al. (2003) Quantitative sensory testing: report of the Therapeutics and Technology Assessment Subcommittee of the American Academy of Neurology. Neurology 60: 898-904

87 Khalili N et al. (2001) Influence of thermode size for detecting heat pain dysfunction in a capsaicin model of epidermal nerve fiber loss. Pain 91: 241-250

88 Facer $\mathrm{P}$ et al. (1998) Correlation of quantitative tests of nerve and target organ dysfunction with skin immunohistology in leprosy. Brain 121: 2239-2247

89 Truini A et al. (2004) Laser evoked potentials for assessing sensory neuropathy in human patients. Neurosci Lett 361: 25-28

90 Chiang HY et al. (2005) Skin denervation, neuropathology, and neuropathic pain in a laserinduced focal neuropathy. Neurobiol Dis 18: 40-53

91 Perretti A et al. (2003) Is Ross syndrome a dysautonomic disorder only? An electrophysiologic and histologic study. Clin Neurophysiol 114: 7-16

92 Granovsky $Y$ et al. (2005) Thermoreceptive innervation of human glabrous and hairy skin: a contact heat evoked potential analysis. Pain 115: 238-247

93 Lombardi R et al. (2005) IgM deposits on skin nerves in anti-myelin-associated glycoprotein neuropathy. Ann Neurol 57: 180-187
94 Lauria G et al. (1998) Neuropathological alterations in diabetic truncal neuropathy: evaluation by skin biopsy. J Neurol Neurosurg Psychiatry 65: 762-766

95 Polydefkis M et al. (2004) The time course of epidermal nerve fibre regeneration: studies in normal controls and in people with diabetes, with and without neuropathy. Brain 127: 1606-1615

96 Toth C et al. (2006) Remote neurotrophic support of epidermal nerve fibres in experimental diabetes. Diabetologia 49: 1081-1088

97 Bianchi R et al. (2006) Protective effect of erythropoietin and its carbamylated derivative in experimental cisplatin peripheral neurotoxicity. Clin Cancer Res 12: 2607-2612

98 Leonelli E et al. (2007) Progesterone and its derivatives are neuroprotective agents in experimental diabetic neuropathy: a multimodal analysis. Neuroscience 144: 1293-1304

99 Schifitto G et al. (2001) Long-term treatment with recombinant nerve growth factor for HIV-associated sensory neuropathy. Neurology 57: 1313-1316

100 Boucek P et al. (2005) Severe depletion of intraepidermal nerve fibers in skin biopsies of pancreas transplant recipients. Transplant Proc 37: 3574-3575

101 Schiffmann R et al. (2006) Enzyme replacement therapy and intraepidermal innervation density in Fabry disease. Muscle Nerve 34: 53-56

102 Jain $\mathrm{M}$ et al. (2000) Histological assessment of dermal nerve damage occurring during multidrug therapy for leprosy. Int J Lepr Other Mycobact Dis 68: 167-171

103 Pareyson D et al. (2006) A multicenter, randomized, double-blind, placebo-controlled trial of long-term ascorbic acid treatment in Charcot-Marie-Tooth disease type 1A (CMT-TRIAAL): the study protocol Pharmacol Res 54: 436-441

104 Kennedy AJ et al. (2005) Pathology and quantitation of cutaneous innervation. In Peripheral Neuropathy, 869-895 (Eds Dyck PJ and Thomas PK) Philadelphia: Elsevier Saunders

\section{Acknowledgments}

Désirée Lie, University of California, Irvine, CA, is the author of and is solely responsible for the content of the learning objectives, questions and answers of the Medscapeaccredited continuing medical education activity associated with this article.

\section{Competing interests} The authors declared no competing interests. 